\title{
Erratum to: Long-term changes in Krill biomass and distribution in the Barents Sea: are the changes mainly related to capelin stock size and temperature conditions?
}

\author{
Elena Eriksen $\cdot$ Padmini Dalpadado
}

Published online: 15 June 2013

(c) Springer-Verlag Berlin Heidelberg 2013

\section{Erratum to: Polar Biology (2011) 34:1399-1409 \\ DOI 10.1007/s00300-011-0995-0}

The historical time series of krill catch data from the Barents Sea from 29 years between 1980 and 2009, which our analysis of long-term changed in krill biomass and distribution was based on, contained (a) three stations with wrong biomass units and (b) 453 stations (equivalent of $5 \%$ of the total of 9,059 stations), for which the start time of trawling was missing.

We have reanalyzed the data series excluding these stations. The removal of data did not alter our conclusion that nighttime catches were significantly higher than daytime catches. Moreover, the quality of nighttime abundance estimates improved. All stations, for which the start time of trawling was not recorded, were erroneously assigned as nighttime stations during data processing in the course of the original analysis. However, the majority of these stations represented very likely daytime catches, since the number of daytime stations generally account for about $2 / 3$ of the total number of stations taken in each year. Therefore, the removal of data resulted in more accurate nighttime abundance estimates, because they are not biased by the confounding impact of mistakenly allocated stations.

In addition, our finding of an inverse relationship between krill and capelin abundances is still valid, in fact, strengthened by the reanalysis. Finally, the result that high krill abundances during 1999-2011 were associated with elevated temperatures was also corroborated, as only the first year (1999) was affected by the omission of erroneous or incomplete station data.

The online version of the original article can be found under doi:10.1007/s00300-011-0995-0.

E. Eriksen $(\bowtie) \cdot$ P. Dalpadado

Institute of Marine Research (IMR), PO Box 1870,

Nordnes, 5817 Bergen, Norway

e-mail: elena.eriksen@imr.no 